\title{
El papel de las ETTs en la reducción del riesgo moral asociado al seguro por accidentes de trabajo: El caso de España
}

\author{
Alfonso Moral de Blas a , Ángel luis Martín-Román a, JuAn Carlos \\ RODRÍGUEZ CABALLERO ${ }^{\mathrm{a}}$ \\ a Universidad de Valladolid, Facultad CC.EE., Avda. del Valle de Esgueva, 6, 47011 Valladolid, \\ España.E-mail: amoral@eco.uva.es, angellm@eco.uva.es, jcrc@eco.uva.es
}

\begin{abstract}
RESUMEN
La presencia de las empresas de trabajo temporal (ETTs) en España es relativamente reciente si se compara con otros países en los cuales éstas tienen más tradición. No obstante, a pesar de su corto recorrido histórico, su funcionalidad en el mercado de trabajo ha ido variando notablemente. En este trabajo se pretende estimar el esfuerzo de los trabajadores contratados por las empresas de trabajo temporal a través del análisis del reporte de cierto tipo de accidentes conocidos dentro de la literatura como accidentes de difícil diagnóstico. Se encuentra evidencia de que los trabajadores contratados a través de una ETT presentan menores niveles de riesgo moral que los trabajadores con contrato indefinido y que los que poseen un contrato temporal directo. Este último resultado puede estar reflejando que las ETTs desempeñan un papel importante en el fomento del esfuerzo de los trabajadores que no puede atribuirse únicamente al hecho de que gestionan contratos laborales de carácter temporal.
\end{abstract}

Palabras clave: Empresas de trabajo temporal, siniestralidad laboral.

\section{The Role of the TWAs in the Reduction of Moral Hazard in Workplace Accident Insurance: The Spanish case}

\begin{abstract}
The presence of temporary work agencies (TWAs) in Spain is relatively recent in comparison with other countries where there is a longer tradition. Nevertheless, despite their short historical existence, their role in the labour market has outstandingly changed. In this paper, we intend to consider the effort of the workers who are hired through TWAs by means of the analysis of a number of accident reports, usually known as hard to diagnose accidents. There are evidences that the workers that are hired through a TWA show lower levels of moral hazard than the ones with a permanent contract and with a direct temporary one. These last results can indicate that TWAs play an important role in promoting the effort of the workers, which can not only be attributed to the fact that they manage temporary contracts.
\end{abstract}

Keywords: Temporary Work Agencies, Labour Accidents.

Clasificación JEL: J28, J22, D81

Artículo recibido en febrero de 2013 y aceptado en junio de 2013

Artículo disponible en versión electrónica en la página www.revista-eea.net, ref. ə-31208

ISSN 1697-5731 (online) - ISSN 1133-3197 (print) 


\section{INTRODUCCIÓN}

En España, desde la reforma laboral de 1994 que supuso la legalización de las empresas de trabajo temporal -en adelante ETTs-, este tipo de empresas han canalizado un creciente volumen de empleo. La literatura económica ha analizado diferentes cuestiones relativas a estas instituciones en relación con el mercado de trabajo, algunas de las cuales se comentarán en una sección posterior. Este trabajo pretende estudiar el papel de las ETTs en la reducción de algunos problemas de riesgo moral asociados al seguro por accidentes de trabajo en España.

El análisis sistemático y desde una perspectiva economicista de los problemas de riesgo moral asociados al seguro de accidentes de trabajo no ha sido un campo de investigación muy prolífico en España hasta épocas recientes, pero sí que goza de gran tradición en Norteamérica. Tanto en Estados Unidos como en Canadá existe ya un volumen considerable de producción científica en este sentido. Resumir toda esta literatura aquí no sería posible, pero una forma muy conveniente de aproximarse a ella es a través de dos trabajos que además establecen una categorización del riesgo moral muy conveniente para los fines de este artículo.

En un primer trabajo, Butler y Worrall (1991) señalan tres tipos de riesgo moral en el que pueden incurrir los trabajadores. En primer lugar estaría lo que los autores llaman riesgo moral de sufrimiento de una lesión. Según esta concepción, los trabajadores toman menos medidas precautorias cuando están cubiertos por un seguro por accidente que cuando no lo están. Esto significa que un aumento de la generosidad en dicho seguro estará asociado a una mayor frecuencia en la siniestralidad laboral. En segundo lugar, estos autores definen el riesgo moral de reporte de una lesión. Este tipo se explica de la siguiente forma: al aumentar la cuantía de la indemnización también aumentan los incentivos de los trabajadores para declarar un accidente, dado un cierto nivel de riesgo. Esto es debido a que la baja laboral está asociada a una mayor renta sustitutiva del salario cuanto más generoso es el seguro. El tercer tipo de riesgo moral lo relacionan directamente con el fraude. En otras palabras, la declaración de accidentes puede aumentar porque al aumentar la cuantía de la indemnización aumentan los incentivos para declarar falsas contingencias laborales.

El segundo trabajo que se va a destacar es el realizado por Fortin y Lanoie (2001). Estos autores amplían el elenco de la tipología del riesgo moral hasta conceptualizar cinco diferentes categorías: (1) el riesgo moral de lesión ex ante; (2) el riesgo moral de causalidad ex ante; (3) el riesgo moral de duración ex post; (4) el riesgo moral de sustitución de seguros y (5) la capacidad discrecional del trabajador para reportar un accidente o no hacerlo. Muy brevemente, cada uno de estos tipos puede explicarse del siguiente modo. En primer lugar, el riesgo moral de lesión ex ante consiste en la toma de menores precauciones por 
parte de los trabajadores debido a que el seguro provee con ingresos al trabajador en caso de accidente. En segundo lugar, riesgo moral de causalidad ex ante que surge porque en ocasiones es difícil identificar que accidentes se han producido realmente en el trabajo. En tercer lugar existe el riesgo moral de duración ex post que provoca que las bajas laborales se prolonguen de forma injustificada. En cuarto lugar está el riesgo moral de sustitución de seguros que puede generar incentivos en los trabajadores para sustituir el seguro por desempleo menos generoso por el de accidente laboral más generoso. Por último un trabajador en ocasiones tiene cierta capacidad discrecional para reportar un accidente y dejar de trabajar o alternativamente seguir trabajando.

Uno de los primeros trabajos empíricos que ha estudiado la relación entre las ETTs y la siniestralidad laboral es el de García Serrano et al., (2010). Los resultados que obtienen indican que los trabajadores de ETTs tienen una probabilidad significativamente menor de sufrir un accidente grave o mortal en comparación con los trabajadores contratados de forma directa (temporales o indefinidos) cuyas características personales y condiciones de trabajo sean idénticas. También encuentran que las duraciones de las bajas son más cortas para los trabajadores de ETTs. Aunque el objetivo de ese trabajo no es el estudio de los problemas de riesgo moral asociados al seguro por accidente de trabajo, los autores explican estos hechos con argumentos que son coherentes con una reducción de lo que aquí se denomina riesgo moral de lesión ex-ante. En otras palabras, se defiende que los trabajadores contratados por ETT reciben cursos formativos específicos de prevención de riesgos laborales que les previenen de los accidentes más graves y si éstos se producen también provoca que las lesiones sean más leves y las duraciones de las bajas más cortas.

El trabajo que aquí se presenta pretende examinar si una institución como la ETT reduce los problemas de riesgo moral de tipo (2) y (5) de la clasificación de Fortin y Lanoie (2001). En otras palabras, se persigue aportar alguna evidencia adicional en relación con la calidad y el esfuerzo de los trabajadores de ETTs pero desde la perspectiva que proporciona el análisis de la siniestralidad laboral. Más precisamente se tratará de estimar el esfuerzo de los trabajadores contratados por las empresas de trabajo temporal mediante el análisis del reporte de cierto tipo de accidentes conocidos dentro de la literatura como accidentes de difícil diagnóstico. El fin de utilizar este tipo de accidentes es que en ellos parece más verosímil que se produzcan episodios de absentismo laboral encubierto. De esta manera se podría obtener una medida del esfuerzo relativo realizado por los trabajadores contratados por empresas de trabajo temporal en relación con los trabajadores contratados de forma directa (bien con contratos temporales, bien con contratos indefinidos).

El resto del trabajo se organiza de la siguiente manera. En el apartado 2 se repasa la literatura previa sobre ETTs y sus efectos en el mercado de trabajo. En 
el apartado 3 se describen las ETTs como empresas de servicios cuya actividad empresarial consiste en el suministro temporal de trabajadores a otras empresas y se reflejan los aspectos esenciales de su regulación en España. En el apartado 4 se presenta la base de datos utilizada y la metodología. Los principales resultados obtenidos se comentan en el apartado 5. Finalmente, la sección 6 resume las principales conclusiones obtenidas en la investigación.

\section{LITERATURA PREVIA}

La forma de contratación laboral atípica que representan las ETTs y los efectos que la actividad de estos intermediarios tiene en el mercado de trabajo han suscitado un gran interés y un importante debate entre los analistas laborales. La mayor parte de la literatura empírica internacional se ha centrado en el análisis de las consecuencias que tiene su actividad tanto para los trabajadores contratados por ellas como para las empresas que utilizan sus servicios.

En relación con las consecuencias para los trabajadores los estudios han investigado si las contrataciones a través de ETTs se caracterizan por índices de precariedad laboral superiores en comparación con otras formas de contratación. Así, los aspectos relacionados con los trabajadores contratados vía ETTs que especialmente han tratado los análisis empíricos son: los niveles salariales (Segal y Sullivan, 1997), la adquisición de formación en el trabajo (Nollen, 1996; Autor, 2001), la movilidad ocupacional y la estabilidad en el empleo (Gottfried, 1991; Pearce, 1998; Segal y Sullivan, 1997; Houseman y Polivka, 2000; Kunda, Barley y Evans, 2002; Autor y Houseman, 2005).

En relación con las consecuencias para las empresas que utilizan los servicios de las ETTs la literatura empírica se ha centrado fundamentalmente en dos cuestiones. La primera se refiere a la mayor flexibilidad laboral que proporciona a las empresas clientes el recurso a las ETTs. Estos intermediarios disponen de información sobre trabajadores potenciales que han pasado previamente por un proceso de selección y pueden conseguir de manera rápida y eficaz el enlace entre las empresas clientes -que necesitan mano de obra en cualquier momento y lugar y durante períodos concretos- y los trabajadores (Houseman, 1997 y 2001, Matusik y Hill, 1998; Gramm y Schnell, 2001, Smith, 2001, Ward et al., 2001). La segunda cuestión es la posible reducción de costes asociada a la utilización de los servicios de las ETTs. Al estar las ETTs especializadas en la selección y el seguimiento de candidatos potenciales pueden desarrollar estas actividades con unos costes medios inferiores a los que tendría que afrontar la empresa cliente si optara por la contratación directa, lo que puede repercutir en una reducción de costes laborales para la última. (Appelbaun y Granrose, 1986; Houseman, 1997, Lautsch, 2002).

Una excelente revisión de la literatura empírica que a nivel internacional ha tratado todos estos aspectos puede encontrarse en el trabajo de Muñóz- Bullón y 
Fernández (2005).

En el caso de España los análisis empíricos que analizan el papel de las ETTs son relativamente escasos y muy recientes, en consonancia con el corto recorrido histórico de estos intermediarios en nuestro mercado de trabajo. Algunos trabajos han abordado desde una perspectiva cuantitativa el papel de las ETTs en la gestión del empleo temporal y su incidencia en las tasas de temporalidad del mercado de trabajo español (Cebrián et al., 2001; Consejo Económico y Social, 2005; Calle Durán et al., 2008). Otros trabajos empíricos analizan aspectos cualitativos más específicos. En particular, una de las cuestiones que ha recibido mayor atención en la literatura empírica sobre ETTs en España es la influencia de la actividad de estos intermediarios en las trayectorias laborales de los trabajadores.

Así, los trabajos de García-Pérez y Muñoz-Bullón (2005a) y de Muñoz-Bullón (2007) analizan la influencia de las ETTs en las transiciones de los trabajadores dentro y fuera del empleo en España durante la década de los noventa. Una de sus conclusiones principales es que, si bien en España las duraciones de los contratos vía ETTs son esencialmente cortas -en torno al 50 por ciento son inferiores al mes (lo que hace que la probabilidad de mantenerse en el empleo de los trabajadores contratados por ETTs sea más baja que en el caso de los individuos que no son contratados por estos intermediarios)- los primeros consiguen salir del paro con mayor facilidad que los segundos, especialmente cuando llevan poco tiempo desempleados. Este hecho afecta de manera más acusada a los trabajadores con niveles de cualificación más bajos y a las mujeres jóvenes, colectivos ambos que presentan las tasas más elevadas de rotación laboral.

Muñoz-Bullón y Rodes (2004) y Amuedo-Dorantes, Malo y Muñoz-Bullón (2005) estudian el efecto de las ETTs sobre la movilidad ocupacional y la estabilidad laboral de los trabajadores contratados vía ETTs frente a los trabajadores que no utilizan esta vía y buscan empleo de forma directa; y concluyen que los primeros tienen mejores expectativas de encontrar un empleo permanente. Estas mejores expectativas son particularmente claras en el caso de los trabajadores con niveles de cualificación elevados (García-Pérez y Muñoz-Bullón, 2005b) y en el caso de las mujeres relativamente jóvenes- de edad igual o inferior a 35 años- para las que las ETTs se convierten en "escalones de acceso" al empleo permanente (Malo y Muñoz-Bullón, 2008). En estos trabajos se mantiene la tesis de que las mejores expectativas laborales (tanto en términos de ascensos laborales como en términos de conseguir empleos permanentes) de los trabajadores contratados vía ETTs se deben al papel de las ETTs como mecanismos de señalización de la calidad de los trabajadores que las utilizan. Las empresas clientes potenciales perciben la experiencia acumulada por los trabajadores de estos intermediarios como un indicador de su esfuerzo y rendimiento futuro. 


\section{NATURALEZA Y REGULACIÓN DE LAS ETTS}

En los países industrializados el negocio de ceder trabajadores a otras empresas se originó en los años cuarenta del siglo pasado cuando las empresas se dieron cuenta de las ventajas de una economía en expansión después de la Segunda Guerra Mundial. A finales de los años 50 la actividad de las empresas de trabajo temporal estaba bien implantada en Estados Unidos, Reino Unido, Holanda y Suiza. Poco después se extendió a otros países de Europa occidental como Bélgica, Francia y República Federal Alemana.

Puede decirse, sin embargo, que el mayor crecimiento de la actividad de las empresas de trabajo temporal tuvo lugar a partir de los años 80 del siglo pasado. Los importantes cambios productivos y tecnológicos producidos durante las últimas décadas del siglo XX generaron grandes cambios en el modelo clásico de producción y de organización de la empresa (modelo fordista). Se trataba de hacer frente a los cambios económicos, institucionales y tecnológicos introduciendo mayores factores de flexibilidad en la producción, gestión y comercialización. En este sentido, las ETTs podían desempeñar un importante papel en la mejora de la flexibilidad empresarial, en especial de la laboral, debido a que constituían una vía para la externalización del trabajo y facilitaban la descentralización productiva, estrategias ambas centrales en las nuevas formas de organización empresarial.

La mayoría de los países occidentales empezaron a legislar sobre la cesión de servicios de trabajadores a partir de los años sesenta, aunque algunos permitieron este tipo de servicios sin establecer una normativa específica al respecto. Entre los que regularon estos servicios y, a pesar de las diferencias entre los sistemas jurídico-laborales a nivel nacional, podría extraerse una pauta común en la finalidad de la regulación: permitir un crecimiento de la gestión que realizan las empresas de trabajo temporal a la vez que mantener la protección para los trabajadores cedidos. ${ }^{1}$

En España, tras el proceso de reforma laboral de los años 1993 y 1994, las ETTs quedaron legalizadas como forma lícita de cesión de trabajadores². En la actualidad las empresas de trabajo temporal se rigen por la Ley 14/1994 que regula las empresas de trabajo temporal, reformada por la Ley 29/1999, y por

\footnotetext{
${ }^{1}$ Un análisis comparado de las legislaciones jurídico-laborales en relación con las empresas de trabajo temporal de los principales países europeos puede encontrase en los documentos Temporary agency work in an enlarge European Union (2006) y Temporary agency work and collective bargaining in the UE (2008), publicados por European Foundation for the Improvement of Living and Working Conditions.

${ }^{2}$ En realidad, desde los años 80 estarían funcionando en España empresas de trabajo temporal, especialmente multinacionales del sector, de modo más o menos paralegal como consultoras de selección o de formación, o como prestadoras de servicios a empresas bajo forma de subcontratas (Albizu, 1997).
} 
otras normas específicas que han regulado aspectos muy concretos relativos a su funcionamiento. Asimismo se rigen también por el Convenio Colectivo aplicable a dichas empresas.

La naturaleza de una empresa de trabajo temporal viene condicionada por su definición legal: son empresas cuya actividad consiste en poner a disposición de otra empresa, denominada usuaria, con carácter temporal, trabajadores por ella contratados, para que puedan hacer frente a necesidades también temporales ${ }^{3}$. La ETT no realiza una función de colocación o de mera puesta en contacto entre una empresa y un trabajador (agencia de colocación), ni tampoco asume la ejecución de la obra o servicio de una empresa con sus propios trabajadores (empresas contratistas o auxiliares), sino que su función específica es la de contratar a trabajadores para cederlos temporalmente a otras empresas.

Las ETT son, por tanto, empresas de servicios cuya actividad empresarial consiste en la cesión temporal de trabajadores a otras empresas. Es esta cesión de los trabajadores lo distintivo de las ETTs con respecto a la relación laboral ordinaria. Aquí se establece una relación triangular en la que la empresa usuaria abona una cantidad a la ETT a cambio de los servicios del trabajador cedido. De hecho, entre la ETT y la empresa usuaria se formaliza un contrato mercantil, llamado contrato de puesta a disposición, y entre la ETT y el trabajador se formaliza un contrato de trabajo.

El contrato de puesta a disposición tiene por objeto la cesión o suministro temporal de mano de obra, teniendo la utilidad para la empresa de trabajo temporal de la percepción de la compensación económica y para la empresa usuaria la disponibilidad de personal externalizando su gestión a través de la contratación temporal "indirecta” frente a la alternativa de la contratación temporal “directa”. El contrato es siempre temporal y su celebración ha de tener siempre una fundamentación causal en la satisfacción de necesidades temporales de mano de obra por parte de la empresa usuaria. En concreto, solamente se puede celebrar para satisfacer necesidades temporales de personal en los cuatro supuestos siguientes, determinados por la legislación ${ }^{4}$ :

- Realización de una obra o servicio determinados.

- Atención de las exigencias circunstanciales del mercado, acumulación de tareas o exceso de pedidos.

- Para sustituir trabajadores de la empresa usuaria con derecho al puesto de

${ }^{3}$ Art. 1 de la Ley 14/1994 por la que se regulan las Empresas de Trabajo Temporal.

${ }^{4}$ Art. 6 de la Ley 14/1994 modificado por Ley 29/1999 y art. 4.1 del Real Decreto 2720/1998 por el que se desarrolla el art. 15 del Estatuto de los Trabajadores. La legislación también señala cuatro supuestos en los que de manera expresa se prohíbe la celebración de contratos de puesta a disposición (Art. 8 de la Ley 14/1994 modificado por Ley 12/2001 de medidas urgentes de reforma del mercado de trabajo para el incremento del empleo y la mejora de su calidad). 
trabajo (enfermedad, vacaciones o excedencia)

- Para cubrir de forma temporal un puesto de trabajo permanente mientras dure el proceso de selección o promoción.

El contrato de trabajo celebrado entre la empresa de trabajo temporal y el trabajador para prestar servicios en la empresa usuaria puede concertarse por tiempo indefinido o por duración determinada coincidente con la del contrato de puesta a disposición ${ }^{5}$. Es por lo tanto la ETT el verdadero empresario del trabajador cedido. Ella es quien le abona su salario y las cotizaciones a la seguridad social y mantiene el poder disciplinario y sancionador sobre él. No obstante, la empresa usuaria responde subsidiariamente de las obligaciones salariales y es además quien, legalmente está autorizada para poner en conocimiento de la ETT los eventuales incumplimientos de los trabajadores cedidos para que adopte las medidas disciplinarias correspondientes. Es decir, el trabajador cedido está sujeto en la práctica a un doble control disciplinario, aunque sólo la ETT pueda sancionarle.

En relación con las condiciones de trabajo aplicables al contrato de trabajo entre ETT y trabajadores cedidos, la regulación de las ETTs establece algunas normas específicas. Entre estas, la regla fundamental se refiere al régimen retributivo, objeto de la reforma llevada a cabo en 1999. Desde esta reforma los trabajadores cedidos y los trabajadores propios de la empresa usuaria perciben la misma remuneración: la retribución total establecida para el puesto de trabajo a desarrollar en el convenio colectivo aplicable a la empresa usuaria, calculada por unidad de tiempo. Junto con la equiparación salarial, los sucesivos convenios colectivos del sector han llevado a cabo una equiparación de otras condiciones de trabajo, especialmente el tiempo de trabajo y su distribución, a los convenios colectivos aplicables a las empresas usuarias.

Las diversas leyes y normas aplicables a las ETTs y el proceso de autorregulación de las mismas que han seguido a la primera ley que las reguló en 1994 han supuesto importantes cambios que han ido sobre todo en la línea de mejorar las condiciones de trabajo de los trabajadores cedidos. Sin embargo, y, a pesar de la evolución legislativa que se ha llevado a cabo en el sector, las ETTs tienen

\footnotetext{
${ }^{5}$ Art. 10 y 11 de la Ley 14/1994, modificados por las leyes 29/1999, 14/2000 de medidas fiscales, administrativas y de orden social y 12/2001 de medidas urgentes de reforma del mercado de trabajo para el incremento del empleo y la mejora de su calidad. A partir de la reforma de la ley de ETTs de 1999 es obligatorio por parte de estas empresas contar con un mínimo de doce trabajadores con contrato indefinido para prestar servicios bajo su dirección-trabajadores de estructura- por cada mil trabajadores contratados anualmente para ser cedidos a empresas usuarias -trabajadores en misión-. Esta obligatoriedad no obedece a un afán de estabilizar la situación laboral de los trabajadores cedidos, pues el legislador tiene claro que la temporalidad es consustancial a la cesión de mano de obra. Lo que persigue es otra cosa: asegurarse de que la ETT disponga de una estructura organizativa suficiente, adecuada y, además, estable.
} 
aún un trato diferencial con respecto a otro tipo de fórmulas de contratación directa. Cabe señalar al respecto los siguientes elementos diferenciadores:

- Las ETTs cuentan con un marco regulatorio específico en materia de prevención de riesgos laborales que, entre otras cosas, permite la cesión de trabajadores sólo y, exclusivamente, en empresas que tengan elaborado su plan de prevención de riesgos laborales ${ }^{6}$.

- El sector de las ETTs es el único sector al que la normativa obliga a destinar el 1 por ciento, más el 0,25 por ciento adicional por Convenio Colectivo, de su masa salarial a formación de los trabajadores cedidos ${ }^{7}$.

- Las ETTs en España tienen limitaciones en cuanto a su actividad. Tienen severas restricciones en la cesión de trabajadores para realizar actividades de especial peligrosidad definidas normativamente (han tenido, hasta la reforma laboral del año 2010 limitaciones en actividades de construcción, actividades de minería, trabajos relacionados con explosivos, etc.) y para trabajar en las Administraciones Públicas ${ }^{8}$.

- Las ETTs están obligadas a pagar una mayor indemnización a sus trabajadores por fin de contrato en relación a los trabajadores con contrato temporal directo (doce días por año trabajado frente a los ocho días que, en general, se establece para los contratos temporales directos) ${ }^{9}$.

- Sobre las ETTs repercute también especialmente el incremento del 36 por ciento en la cuota empresarial por contingencias comunes que recae sobre los contratos de menos de 7 días (que suponen en torno al 30 por ciento de los celebrados por estas empresas) ${ }^{10}$.

Para finalizar este apartado y a modo de conclusión, cabe señalar que aunque la legislación española limitó, hasta la reciente reforma laboral del año $2012^{11}$,

${ }^{6}$ Real Decreto 216/1999, sobre disposiciones mínimas de seguridad y salud en el trabajo en el ámbito de las empresas de trabajo temporal.

${ }^{7}$ Art. 12 Ley 14/1994, modificado por Ley 29/1999.

${ }^{8}$ No obstante, en el art. 17.6, apartado 2 de la Ley 35/2010, de medidas urgentes para la reforma del mercado de trabajo, se establece que mediante acuerdos interprofesionales o convenios colectivos podrán determinarse, por razones de seguridad y salud en el trabajo, limitaciones para la celebración de contratos de puesta a disposición en las actividades de la construcción, la minería a cielo abierto, las industrias extractivas por sondeos en superficie terrestre y en los trabajos relacionados con explosivos.

${ }^{9}$ Art. 11 Ley 14/1994 modificado por Ley 29/1999.

${ }^{10}$ Introducido por Ley 12/2001 de medidas urgentes de reforma del mercado de trabajo para el incremento del empleo y la mejora de su calidad

${ }^{11}$ La Ley 3/2012 de medidas urgentes para la reforma del mercado laboral ha introducido la posibilidad de que las Empresas de Trabajo Temporal actúen también como agencias de colocación (art. 1, Ley 3/2012). 
la función de las ETTs a facilitar trabajadores temporales a las empresas clientes con el fin de satisfacer sus necesidades temporales de mano de obra (además con severas limitaciones en relación con los sectores productivos y ocupaciones en las que pueden intervenir), la realidad ha mostrado que estas empresas han venido realizando actividades cada vez más profesionalizadas como son la intermediación en el mercado de trabajo, la selección del trabajador idóneo y la formación profesional y de prevención de los trabajadores contratados.

\section{DATOS Y METODOLOGÍA}

\subsection{Base de datos}

Los datos empleados en este trabajo proceden de la Estadística de Accidentes de Trabajo (EAT). Se consideran los datos del año 2006 y del año 2011 (último año disponible) al objeto de evaluar la posible influencia de la crisis económica y laboral que comenzó en el año 2008 en el comportamiento diferencial de diferentes colectivos de trabajadores en lo relativo a la siniestralidad laboral.

La EAT proporciona información registral de todos los accidentes laborales que se han producido en España cada año y que han supuesto, al menos, un día de baja laboral. Los datos que ofrece se obtienen de la información contenida en los partes de alta y baja que se remiten al Ministerio de empleo y Seguridad Social. Estos documentos fueron establecidos por Orden TAS / 2926/2002, de 19 de noviembre, en la que se establecen nuevos modelos para la notificación de accidentes de trabajo y se posibilita su transmisión por procedimiento electrónico mediante el Sistema Delt@ ${ }^{12}$.

Los datos brutos se han depurado de diversas formas. En primer lugar se eliminan todos aquellos problemas de registro asociados a las variables que posteriormente se utilizarán en el análisis econométrico. También se han eliminado los datos pertenecientes a las ciudades autónomas de Ceuta y Melilla. Se suprimen las ramas de actividad donde las ETT tienen un peso reducido como Extracción y Coquerías o Administraciones Públicas y Organismos Internacionales. Finalmente, se eliminan los trabajadores autónomos y aquellos pertenecientes a ETT que además figuran como poseedores de un contrato indefinido. De esta manera se intenta medir de una forma más nítida el efecto puramente contractual. Una vez hechas todas las depuraciones en la base de datos de 2006 se mantienen 725.636 datos de accidentes laborales, de los cuales 22.481 se refieren a trabajadores de empresas de trabajo temporal (un 3,1\%). En la base

\footnotetext{
${ }^{12}$ País Vasco y Cataluña han establecido sus propios sistemas de transmisión electrónica, si bien Cataluña también trasmite las notificaciones de accidentes al sistema Delt@, aunque a efectos de elaboración de la estadística, para estas dos comunidades se utilizan ficheros externos a Delt@
} 
correspondiente a 2011 se mantienen 439.077, de los cuales 8.069 se refieren a trabajadores de empresas de trabajo temporal (un $1.9 \%)^{13}$

\subsection{Metodología}

La estrategia empírica seguida en este trabajo consiste en explotar la diferente composición de los accidentes laborales. Más precisamente se estudia la frecuencia relativa con la que se producen los denominados accidentes de difícil diagnóstico o de fácil ocultación.

Para llevarla a cabo se construye una variable dependiente que indica la probabilidad de que el accidente sufrido pertenezca al grupo de los conocidos dentro de la literatura como de difícil diagnóstico (dislocaciones, esguinces y torceduras de acuerdo a la clasificación de la EAT a partir del año 2003). Esta forma de proceder utiliza la misma variable dependiente que los trabajos de Campolietti y Hyatt (2006), Martín-Román y Moral (2008) y Moral et al., (2010). Esta selección de los trabajadores accidentados se justifica puesto que la literatura previa ha establecido con claridad que los comportamientos estratégicos de los individuos asociados a cuestiones de riesgo moral se producen con más frecuencia en los accidentes de este tipo (Smith, 1990; Butler et al., 1996; Card y McCall, 1996; Bolduc et al., 2001). Así pues, la variable dependiente empleada toma el valor el valor 1 si el accidente es de difícil diagnóstico y cero en el caso contrario.

Se trata, por lo tanto, de la estimación de una probabilidad condicionada. Es decir, estudiamos el peso de los accidentes de difícil diagnóstico dentro del total de accidentes reportados. El interés de la investigación reside en analizar las diferencias en la composición de los accidentes que han ocasionado una baja laboral y no en el cálculo de la incidencia de la siniestralidad en el puesto de trabajo $^{14}$. Para este objetivo se dispone de todo el universo de trabajadores accidentados y por ello las conclusiones pueden extrapolarse a ese colectivo y no al total de trabajadores.

En resumen, se examina la composición de accidentes laborales, no la inci-

\footnotetext{
${ }^{13}$ Los informes anuales de accidentes de trabajo en España proporcionados por el Instituto Nacional de Seguridad e Higiene en el Trabajo (INSHT) entre los años 2006 y 2011 ponen de manifiesto que el número total de accidentes laborales ha ido disminuyendo paulatinamente con el paso del tiempo. Si únicamente nos quedásemos con este dato, se podría argumentar que el principal causante de esta disminución es la crisis económica actual, que ha supuesto un importante incremento de la población desempleada. No obstante, estos informes indican que el índice de incidencia (número total de accidentes laborales por cada 100.000 trabajadores con contingencias de accidente laboral y enfermedad profesional cubiertas) también han ido disminuyendo durante este período. http://www.oect.es/portal/site/Observatorio/

${ }^{14}$ La incidencia absoluta recogería la probabilidad de que un trabajador reporte un accidente de difícil diagnóstico.
} 
dencia absoluta, para el colectivo de trabajadores accidentados, no para el global de población ocupada. Dicho esto, la composición puede verse influenciada por diversas características poblacionales u ocupacionales; sin embargo estos efectos se tienen en cuenta incluyendo diferentes variables de control en la especificación econométrica.

Primero se realiza un análisis probit de los datos y a continuación se plantea una generalización de la descomposición de Oaxaca Blinder para modelos no lineales. Con esta descomposición se consigue determinar que parte de la diferencia observada entre el porcentaje de accidentes de difícil diagnóstico asociada a cada tipo de contrato se justifica por que los grupos son diferentes, y que parte es consecuencia de una respuesta diferente a características similares. El tamaño de esta segunda parte (el componente injustificado de la descomposición) será un indicador de la intensidad relativa con la que se produce el riesgo moral y-o situaciones relacionadas con comportamientos oportunistas o estratégicos de los trabajadores. Finalmente se identificará la parte de esos componentes que se asocia a cada grupo de variables.

En los trabajos de Oaxaca (1973) y Blinder (1973) se desarrolla un método para analizar las diferencias entre grupos poblacionales (la denominada descomposición de Oaxaca-Blinder) que se ha mostrado muy productivo dentro del campo de investigación de la Economía Laboral, en especial en los temas referidos a la discriminación salarial.

Sin embargo, la descomposición de Oaxaca-Blinder presenta dos limitaciones fundamentales. En primer lugar, se concibió originalmente para el análisis de modelos lineales. En segundo lugar, plantea problemas de identificación si se intenta calcular la aportación de variables individuales en presencia de grupos de dummies (Oaxaca y Ransom, 1999). El análisis econométrico empleado en este trabajo sigue el planteamiento de Yun $(2004,2005)$ que supera las dos limitaciones anteriores. Se trata de una generalización de la descomposición de Oaxaca-Blinder para modelos no lineales que resuelve el problema de identificación en la descomposición detallada.

De acuerdo con Yun, si la variable Y es función de una combinación de variables independientes $\mathrm{XB}$ a través de una función no lineal $\varphi(Y=\varphi(X B))$. La diferencia entre el valor medio de la variable dependiente entre dos grupos poblacionales 1 y 2 , puede descomponerse como sigue:

$$
\overline{Y_{1}}-\overline{Y_{2}}=\left[\overline{\varphi\left(X_{1} \beta_{1}\right)}-\overline{\varphi\left(X_{2} \beta_{1}\right)}\right]+\left[\overline{\varphi\left(X_{2} \beta_{1}\right)}-\overline{\varphi\left(X_{2} \beta_{2}\right)}\right]
$$

El primer sumando de la parte derecha de la expresión (1) recoge la diferencia justificada por el distinto valor que toman los regresores en cada grupo. El segundo término reflejará la diferente repercusión de características similares. A partir de esa descomposición agregada, Yun propone un método para obtener la contribución de cada variable a partir de la siguiente expresión: 


$$
\overline{Y_{1}}-\overline{Y_{2}}=\sum_{i=1}^{T} W_{\Delta X}^{i}\left[\overline{\varphi\left(X_{1} \beta_{1}\right)}-\overline{\varphi\left(X_{2} \beta_{1}\right)}\right]+\sum_{i=1}^{T} W_{\Delta \beta}^{i}\left[\overline{\varphi\left(X_{2} \beta_{1}\right)}-\overline{\varphi\left(X_{2} \beta_{2}\right)}\right]
$$

donde, $W_{\Delta X}^{i}=\frac{\left(\overline{X_{1}^{i}}-\overline{X_{2}^{\imath}}\right) \beta_{1}^{i}}{\sum_{i=1}^{T}\left(\overline{X_{1}^{i}}-\overline{X_{2}^{i}}\right) \beta_{1}^{i}} ; W_{\Delta \beta}^{i}=\frac{\overline{X_{2}^{\imath}}\left(\beta_{1}^{i}-\beta_{2}^{i}\right)}{\sum_{i=1}^{T} \overline{X_{2}^{i}}\left(\beta_{1}^{i}-\beta_{2}^{i}\right)} \sum_{i=1}^{T} W_{\Delta X}^{i}=\sum_{i=1}^{T} W_{\Delta \beta}^{i}=1$, y $\mathrm{T}$ es el número de variables.

Esta descomposición detallada, cuando se introducen grupos de variables ficticias, presenta un problema de identificación que obliga a realizar una regresión normalizada. Esta normalización puede consultarse en el trabajo de Yun (2005). Siguiendo el trabajo de Corrales et al., (2008), es el segundo componente (el denominado componente injustificado) el que se va tomar como un índice de la intensidad relativa con el que se produce el riesgo moral. En otras palabras, el hecho de que idénticas características afecten de forma diferente a grupos "estadísticamente" similares se interpreta en este trabajo como un indicador del comportamiento oportunista y/o estratégico de los trabajadores

\section{RESULTADOS}

La observación de los datos desde un punto de vista meramente descriptivo pone de manifiesto que la probabilidad de sufrir un accidente de difícil diagnóstico es menor en los trabajadores con contrato temporal directo que en el caso de los trabajadores con contrato indefinido y aún más reducido en el caso de los que tienen un contrato temporal a través de una ETT. Las Tablas A1 y A2 del apéndice en las que se presenta un resumen descriptivo de las variables utilizadas en el análisis para los años 2006 y 2011 así lo reflejan. En el año 2006 el $45,1 \%$ de los accidentes que declaran los trabajadores indefinidos son de difícil diagnóstico mientras que este porcentaje es del $41,1 \%$ en los temporales directos y del 38,1\% en las ETTs. En 2011 estos porcentajes son el 51,4\%, el $48,95 \%$ y el $44,1 \%$ respectivamente. También dejan patente que las diferencias entre las diferentes formas de contratación pueden ser consecuencia de las características personales de los trabajadores, de las ocupaciones que desempeñan o de la rama de actividad a la que pertenecen. Para ambos años en las ETTs hay un mayor porcentaje de accidentados inmigrantes y varones, los trabajadores son más jóvenes y tienen menos experiencia. Además, los accidentes de las ETTs se concentran especialmente en las ramas de actividad referidas a manufacturas y en las ocupaciones menos cualificadas.

Estas diferencias justifican la necesidad de un estudio pormenorizado para analizar su origen y para determinar en qué medida son consecuencia de un mero efecto contractual asociado al comportamiento de los trabajadores.

En la Tabla 1 y en las Tablas A3 y A4 del Apéndice 1 se muestran los resultados de la estimación tipo probit con la que se inicia el trabajo econométrico. En esa tabla se presenta el valor de los coeficientes de las 
variables (efectos marginales) que recogen si el trabajador tiene un contrato temporal directo y si lo tiene indefinido. La estimación se realiza para dos modelos, uno sin controles (modelo I), y otro que controla por las características personales, las características del accidente, la ocupación y la rama de actividad, el tamaño de la empresa y la Comunidad Autónoma (modelo II). Se sigue que el coeficiente indica la diferencia de probabilidad de sufrir un accidente de los denominados de difícil diagnóstico de este tipo de trabajadores en relación con los que son contratados a través de ETT.

Los resultados revelan que, una vez que el accidente se ha declarado, es más probable que la lesión sea de difícil diagnóstico si el trabajador es indefinido o tiene un contrato temporal directo que si es contratado a través de una ETT. Los resultados detallados de estas estimaciones aparecen en las Tablas A3 y A4 del Apéndice 1.

Tabla 1

Resultados de la estimación de la probabilidad de que el accidente sufrido ocasione una lesión de difícil diagnóstico. Años 2006 y 2011

\begin{tabular}{|c|c|c|c|c|}
\hline \multirow{2}{*}{$\begin{array}{c}\text { Año } \\
\text { Modelo }\end{array}$} & \multicolumn{2}{|c|}{2006} & \multicolumn{2}{|c|}{2011} \\
\hline & $\mathbf{I}$ & II & $\mathbf{I}$ & II \\
\hline Temporal (no ETT) & $\begin{array}{c}0.029 \\
(0.003)\end{array}$ & $\begin{array}{c}0.027 \\
(0.004)\end{array}$ & $\begin{array}{c}0.044 \\
(0.006)\end{array}$ & $\begin{array}{r}0.019 \\
(0.006)\end{array}$ \\
\hline Indefinido & $\begin{array}{l}0.069 \\
(0.003)\end{array}$ & $\begin{array}{l}0.048 \\
(0.004)\end{array}$ & $\begin{array}{c}0.074 \\
(0.006)\end{array}$ & $\begin{array}{c}0.041 \\
(0.006)\end{array}$ \\
\hline Características Personales $^{1}$ & No & $\mathrm{Si}$ & No & $\mathrm{Si}$ \\
\hline $\begin{array}{l}\text { Características del } \\
\text { Accidente }^{2}\end{array}$ & No & $\mathrm{Si}$ & No & $\mathrm{Si}$ \\
\hline Código de Ocupación ${ }^{3}$ & No & $\mathrm{Si}$ & No & $\mathrm{Si}$ \\
\hline Código de Actividad ${ }^{4}$ & No & $\mathrm{Si}$ & No & $\mathrm{Si}$ \\
\hline Comunidad Autónoma $^{5}$ & No & $\mathrm{Si}$ & No & $\mathrm{Si}$ \\
\hline Tamaño de la Empresa $^{6}$ & No & $\mathrm{Si}$ & No & $\mathrm{Si}$ \\
\hline
\end{tabular}

Notas: La variable dependiente es una variable dummy que toma el valor 1 en el caso de que el accidente sea de difícil diagnóstico. La tabla presenta los efectos marginales del tipo de contrato. Los errores estándar están entre paréntesis.

${ }^{1}$ Características personales: Género, edad, edad al cuadrado, antigüedad, antigüedad al cuadrado, y dos variables que indican si el trabajador accidentado es inmigrante de país desarrollado o no desarrollado.

${ }^{2}$ Características del accidente: Variables que indican si el accidente se produce en lunes o el fin de semana, el lugar donde se ha producido, las horas que se llevaban trabajadas y el turno de trabajo.

${ }^{3}$ Código de ocupación: Ocho dummies de ocupación donde la referencia son las ocupaciones correspondientes a directores y científicos.

${ }^{4}$ Código de actividad: Ocho dummies de rama de actividad donde la referencia son las actividades referidas al sector primario.

${ }^{5}$ Comunidad Autónoma: Diecisiete dummies donde la referencia es Andalucía.

${ }^{6}$ Tamaño de la empresa. Siete dummies donde la referencia son las empresas de menos de 10 trabajadores.

Fuente: Elaboración propia a partir de los datos de la EAT. 
Para corroborar los resultados anteriores se realiza un análisis de descomposición con el fin de identificar, qué parte de las diferencias es consecuencia de las distintas características recogidas por los regresores del modelo (componente justificado), y que parte se debe a que características similares tienen efectos distintos en función del grupo poblacional analizado (componente injustificado).

Los resultados de la descomposición realizada de acuerdo a la ecuación (1) para los años 2006 y 2011 se recogen en la Tabla 2. En la primera columna aparece la diferencia observada en tanto por uno entre el porcentaje de accidentes de difícil diagnóstico que reporta cada tipo de contrato. La segunda y la tercera fila muestran la parte justificada e injustificada de esta diferencia en términos absolutos. Por su parte la cuarta y quinta filas presentan los pesos porcentuales de cada componente. Los valores de esta tabla ponen de manifiesto que con los datos del año 2006 las diferencias en el porcentaje de lesiones de difícil diagnóstico reportadas por los diferentes trabajadores, en su mayor parte, no se explican por las características de las variables incluidas en la regresión.

\section{Tabla 2}

Descomposición de la diferencia en la probabilidad de sufrir un accidente de difícil diagnóstico según la relación contractual de los accidentados

\begin{tabular}{c|l|c|cc|cc}
\hline & & Diferencia (1) & Justificado (2) & Injustificado (3) & $\mathbf{( 2 / 1 )} \%$ & $\mathbf{( 3 / 1 )} \%$ \\
\hline \multirow{2}{*}{2006} & Temporal / ETT & 0.029 & 0.003 & 0.026 & $10.3 \%$ & $89.7 \%$ \\
\cline { 2 - 7 } & Indefinido I ETT & 0.069 & 0.017 & 0.052 & $24.6 \%$ & $75.4 \%$ \\
\hline \multirow{2}{*}{2011} & Temporal / ETT & 0.044 & 0.036 & 0.008 & $81.5 \%$ & $18.5 \%$ \\
\cline { 2 - 7 } & Indefinido I ETT & 0.073 & 0.032 & 0.041 & $43.6 \%$ & $56.4 \%$ \\
\hline
\end{tabular}

Nota: En las Tablas A3 y A4 del apéndice 1 se recogen los resultados de las estimaciones empleadas para esta descomposición agregada.

Fuente: Elaboración propia a partir de los datos de la EAT.

El resultado más destacable se obtiene al comparar los accidentados con contrato temporal directo y los pertenecientes a una ETT. Las características diferentes de cada colectivo de trabajadores explica solamente un 10,3\% de las diferencias observadas en el reporte de accidentes de difícil diagnóstico. El $89,7 \%$ restante parece responder a un efecto puramente contractual que lleva a que los trabajadores contratados a través de ETTs, quizá debido al doble control disciplinario al que se encuentran sometidos, reporten con menor intensidad accidentes de difícil diagnóstico.

Los resultados obtenidos con los datos del año 2011 reflejan una disminución importante en los componentes injustificados, tanto en el caso de que se comparen los trabajadores indefinidos con los trabajadores temporales de ETTs, como en el caso de que se comparen los dos colectivos de trabajadores tempo- 
rales. Esta disminución es mucho más acusada en el segundo de estos casos en el que la parte de la diferencia observada que no explican las características diferentes de ambos colectivos solamente representa el 18,5\%. Puede interpretarse como una reducción de la intensidad con la que se produce el riesgo moral y-o las situaciones relacionadas con comportamientos oportunistas o estratégicos de los trabajadores. La crisis económica y laboral que sufre nuestro país desde el año 2008 ha llevado a que los trabajadores, sobre todo los temporales, se preocupen más por mantener el empleo lo que en gran medida puede explicar la disminución de la aportación del componente injustificado a la diferencia observada en el reporte de accidentes de difícil diagnóstico.

La última parte de este análisis consiste en la realización de la descomposición detallada de acuerdo con la ecuación (2). El objetivo de este nuevo análisis es determinar el peso de las diferentes características analizadas a la hora de explicar los distintos componentes. Los resultados de dicha descomposición detallada para el año 2011 se muestran en la Tabla $3^{15}$. Las primeras dos columnas de la Tabla 3 muestran descomposición detallada de la diferencia existente entre el porcentaje de accidentes de difícil diagnóstico que reportan los trabajadores temporales directos cuando se les compara con los de las ETTs. Como ya se podía observar en la Tabla 2, la mayor parte de la diferencia se debía al componente justificado, pero ahora podemos saber qué características son las que más influyen. Así se comprueba que casi el 100\% de la diferencia observada es consecuencia de que los trabajadores de las Empresas de Trabajo Temporal realizan actividades económicas diferentes y seguramente, menos propensas a presentar este tipo de lesiones de difícil diagnóstico. Este resultado es coherente con el que ya se observaba en las estimaciones probit iniciales. También se aprecia que las características personales y del accidente explican una parte importante de estas diferencias, mientras que los distintos tamaños de empresa reflejarían diferencias en el sentido contrario. En cuanto al componente injustificado, se puede comprobar que la parte más explicativa viene del diferente efecto que tienen las características personales en cada grupo de accidentados.

Por su parte, las columnas tres y cuatro de la Tabla 3 muestran la descomposición detallada de las diferencias observadas en el porcentaje de accidentes de difícil diagnóstico que reportan los trabajadores indefinidos en relación con los de las ETTs. Nuevamente los resultados son coherentes con lo recogido en la Tabla 1 ya que la mayor parte del componente justificado se debe a las características personales y a las actividades que desarrolla cada trabajador. En cuanto al componente injustificado, se corrobora el hecho de que, en su mayor parte, es consecuencia de que las características personales afectan más al grupo de trabajadores indefinidos. ${ }^{15}$ No se incluyen los resultados de la descomposición detallada con los datos de accidentes del
año 2006 para no hacer excesivamente prolija la presentación de resultados. 
Tabla 3

Descomposición detallada de la diferencia en la probabilidad de sufrir un accidente de difícil diagnóstico según la relación contractual de los accidentados

\begin{tabular}{l|cc|cc}
\hline & \multicolumn{2}{|c|}{ Temporal - ETT } & \multicolumn{2}{c}{ Indefinido - ETT } \\
\cline { 2 - 4 } & Justificado & Injustificado & Justificado & Injustificado \\
\hline Características personales & 0.0116 & 0.1786 & 0.0163 & 0.2325 \\
Características del accidente & -0.0006 & -0.0100 & 0.0013 & 0.0044 \\
C.N.O. & 0.0014 & -0.0294 & 0.0001 & -0.0296 \\
C.N.A.E. & 0.0316 & -0.0128 & 0.0171 & -0.0037 \\
Tamaño de la empresa & -0.0040 & -0.0054 & -0.0025 & -0.0091 \\
Comunidad Autónoma & -0.0042 & -0.0344 & 0.0002 & -0.0358 \\
Constante & 0 & -0.0785 & 0 & -0.1085 \\
\hline TOTAL & $\mathbf{0 . 0 3 5 8}$ & $\mathbf{0 . 0 0 8 2}$ & $\mathbf{0 . 0 3 2 0}$ & $\mathbf{0 . 0 4 1 4}$ \\
\hline
\end{tabular}

Nota: En la Tabla A4 del apéndice 1 se recogen los resultados de las estimaciones empleadas para esta descomposición detallada.

Fuente: Elaboración propia a partir de datos de la EAT.

\section{CONCLUSIONES}

Los trabajadores contratados a través de una ETT presentan un comportamiento diferente al resto de los trabajadores en lo relativo a la siniestralidad laboral. El trabajo de García Serrano et al., (2010) encuentra que los trabajadores de ETTs experimentan accidentes menos graves y las duraciones de las bajas son más cortas. Aunque el objetivo de ese trabajo no es el estudio de los problemas de riesgo moral asociados al seguro por accidente de trabajo, los autores explican estos hechos con argumentos que son coherentes con una reducción de lo aquí se denomina riesgo moral de lesión ex-ante.

En el presente trabajo se ha pretendido profundizar en esta cuestión. Para ello, se toma un índice de incidencia relativo (la probabilidad de sufrir un accidente de los denominados de difícil diagnóstico condicionado a haber sufrido un accidente) y se obtienen las diferencias observadas entre distintos colectivos de trabajadores (indefinidos, con contrato temporal “directo” y contratados temporalmente vía ETTs). El análisis econométrico de descomposición que se realiza posteriormente permite identificar que parte de estas diferencias es debida a las características diferentes de los colectivos y que parte se debe a que características similares tiene efectos distintos según el colectivo. Esta última se interpreta como una medida de la intensidad relativa con la que se producen situaciones de riesgo moral y-o relacionadas con comportamientos oportunistas o estratégicos de los trabajadores a la hora de declarar un accidente de fácil ocultación.

Los resultados obtenidos muestran que los trabajadores de ETTs presentan con menor intensidad las situaciones mencionadas en el párrafo anterior. El comportamiento diferencial de este colectivo de trabajadores se manifiesta de 
manera muy acusada en un año de prosperidad económica. El doble control disciplinario al que están sujetos los trabajadores de empresas de trabajo temporal (de la empresa usuaria y de la propia empresa de trabajo temporal), puede, en gran medida, explicar el menor reporte relativo de accidentes de fácil ocultación por parte de este colectivo.

No obstante, en momentos de recesión económica y de fuerte aumento del desempleo como el que vive nuestro país desde el año 2008, cabe pensar que los trabajadores, sobre todo los trabajadores con contrato temporal, estén más preocupados por mantener su empleo lo que explicaría la importante reducción de las diferencias injustificadas en el reporte de accidentes de difícil diagnóstico.

\section{REFERENCIAS BIBLIOGRÁFICAS}

ALBIZU, E. (1997). Flexibilidad Laboral y Gestión de los Recursos Humanos. Ariel. Barcelona.

AMUEDO-DORANTES, C., MALO, M.A. y MUÑOZ-BULLÓN (2005). "Empresas de Trabajo temporal y trayectoria laboral.". Informe final 2005. Cátedra AGGETT- Universidad de Salamanca.

APPELBAUN, E y GRANROSE, C. (1986). "The Efficiency of Temporary Help and Part-time Employment". Personal Administrator, Enero, 71-83.

AUTOR, D. H. (2001). "Why Do Temporary Help Firms Provide Free General Kills Training?". Quarterly Journal of Economics, 116 (4), 1409-1448.

AUTOR, D. H. y HOUSEMAN, S.N. (2005). "Do the temporary help jobs improve labour market outcomes for low-skilled workers? Evidence from radom assigments". Upjohn Institute Staff Working Paper, nº 05-124.

BLINDER, A. S. (1973). "Wage discrimination: reduced form and structural estimates". Journal of Human Resources, 8, 436-455.

BOLDUC, B., FORTIN, B., LABRECQUE, F. y LANOIE P. (2001). "Workers' compensation, moral hazard, and the composition of workplace injuries". Journal of Human Resources, 37(3), 623-652.

BUTLER, R. J. y WORRALL, J. D. (1991). "Claims reporting and risk bearing moral hazard in workers' compensation". Journal of Risk and Insurance, 63, 191-204.

BUTLER, R. J., DURBIN, D. L. y HELVACIAN, N. M. (1996). "Increasing claims for soft tissue injuries in workers' compensation: cost shifting and moral hazard". Journal of Risk and Uncertainty, 13, 73-87.

CALLE DURÁN, M.C., ORTIZ DE URBINA, M. y ROMERO, M. (2008). "La gestión de la temporalidad. El papel de las empresas de trabajo temporal". Boletín Económico de ICE, n² 2942, 39-52. 
CAMPOLIETI, M. y HYATT, D. E. (2006). "Further evidence on the "Monday effect" in workers' compensation". Industrial and Labor Relations Review, 59(3), 438-450.

CARD, D. y MCCALL, B. P. (1996). "Is workers' compensation covering uninsured medical cost? Evidence from the "Monday effect". Industrial and Labor Relations Review, 49(4), 690-706.

CEBRIÁN, I., FELgueroso, F., MORENO, G. y TOHARIA, L. (2001). "Un análisis Económico de las empresas de trabajo temporal". En Ricardo Escudero, dir. Empresas de Trabajo Temporal - Jornadas de debate sobre empresas de trabajo temporal, Madrid, Consejo Económico y Social de la Comunidad de Madrid, 19-52.

CONSEJO ECONÓMICO Y SOCIAL (2005). "Una década de empresas de trabajo temporal". Boletín del Observatorio de Relaciones Industriales, $n^{\circ}$ 81, mayo. Consejo Económico y Social. Madrid, 4

CORRALES, H., MARTÍN-ROMÁN, A. y MORAL, A. (2008). "La duración de las bajas por accidente laboral en España: ¿Se justifican las diferencias entre comunidades autónomas?". Revista de Economía Laboral, 5, 73-98.

EUROPEAN FOUNDATION FOR THE IMPROVEMENT OF LIVING AND WORKING CONDITIONS (2006). Temporary agency work in an enlarged European Union.

EUROPEAN FOUNDATION FOR THE IMPROVEMENT OF LIVING AND WORKING CONDITIONS (2008). Temporary agency work and collective bargaining in the UE.

FORTIN, B. y LANOIE, P. (2001). "'Incentive effects of workers' compensation insurance: a survey"'. Handbook of Insurance, Springer, 421-458.

GARCÍA-PÉREZ, J. I. y MUÑOZ-BULLÓN, F. (2005a). "Are Temporary Help Agencies Changing Mobility Patterns in the Spanish Labour Market?". Spanish Economic Review vol. 7, nº. 1, 43-65.

GARCÍA-PÉREZ, J. I. y MUÑOZ-BULLÓN, F. (2005b). "Temporary Help Agencies and Occupational Mobility". Oxford Bulletin of Economics and Statistics, vol. 67, nº. 2, 163-180.

GARCÍA SERRANO, C. HERNÁNZ, V. y TOHARIA, L. (2010). "Mind the gap, please! The effect of temporary help agencies on the consequences of work accidents". Journal of Labour Research n³1, 162-182

GOTTFRIED, H. (1991). "Mechanisms of Control in the Temporary Help Service Industry". Sociological Forum, 6(4), 699-713.

GRAMM, C. L. y SCHNELL, J.F. (2001). "The Use of Flexible Staffing Arrangements in Core Production Jobs". Industrial and Labor Relations Review, 54(2), 245-258.

HOUSEMAN, S. N. (1997). "Temporary, Part-time and Contract Employment in the United States: New Evidence from an Employer Survey". Kalamazoo, Mich. W.E. Upjohn Institute for Employment Research. 
HOUSEMAN, S. N. (2001). "Why employers use flexible staffing arrangements: evidence from an establisment survey". Industrial and Labor Relations Review, 55, 149-70.

HOUSEMAN, S. N. y POLIVKA, A. (2000). "The implications of flexible staffing arrangements for job stability", en On the job: Is long-term employment a thing of the past?. En David Neumark (ed.), New York: Russell Sage Foundation, 427-62.

KUNDA, G., BARLEY, S.R. y EVANS, J. (2002). "Why do Contractors Contract? The experience of Highly Skilled Technicals Professionals in a Contingent Labor Market". Industrial and Labor Relations Review, 55 (2), 234261.

LAUTSCH, B.A. (2002). "Uncovering and Explaining Variance in the Features and Outcomes of Contingent Work within Firms". Industrial and Labor Relations Review. 56 (1), 23-43.

MALO, M.A. y MUÑOZ-BULLÓN, F. (2008). "Temporary Help Agencies and Participation Histories in the Labour Market: A Sequence-Oriented Approach". Estadística Española, vol. 50, nº. 167, 21-61.

MARTÍN-ROMÁN, A. y MORAL, A. (2008). "Moral Hazard and Gender Differences in the Workplace Accidents Insurance". Empirical Economic Letters, 7(7), 707-713.

MORAL, A. MARTÍN-ROMÁN, A. y RODRÍGUEZ, J.C. (2010). "La antigüedad y las diferencias de esfuerzo entre trabajadores de distintas zonas geográficas: un estudio de los accidentes de trabajo". Estudios de Economía Aplicada, 28(1). 1-20

MATUSIK, S. F. y HIILL, C.W.L. (1998). "The Utilization of Contingent Work, Knowledge, and Competitive Advantage". Academy of Management Review, 23(4), 680-97.

MUÑOZ-BULLÓN, F. y RODES, E. (2004). "Temporalidad y Señalización en el Mercado de Trabajo: El Papel de las Empresas de Trabajo Temporal". Cuadernos de Economía y Dirección de la Empresa, vol. 18, 35-67.

MUÑOZ-BULLÓN, F. y FERNÁNDEZ, I. (2005). "Las Empresas de Trabajo Temporal". En J. Bonache y A. Cabrera, Dirección de Personas. Ed. Prentice Hall, Madrid, 427-462.

MUÑOZ-BULLÓN, F. (2007). Empresas de Trabajo Temporal y Abandono del Desempleo. Ed. Thomson-Civitas.

NOLLEN, S. D. (1996). "Negative Aspects Of Temporary Employment". Journal of Labor Research, 17(4), 567-582.

OAXACA, R. y RANSOM, M. (1999). "Identification in detailed wage decompositions". Review of Economics and Statistics, 81:1, 154-157.

OAXACA, R. (1973). "Male-female wage differentials in urban labour markets". International Economic Review, 14, 693-709.

PEARCE, J.L. (1998). "Job Security is important, but not for the reasons you might think: the example of contingent workers". En Cooper, C.L. y Rous- 
seau, D.M. (ed.): Trends in Organizational Behavour, 5. Chichester, U.K. Wiley, 31-46.

SEGAL, L. M. y SULLIVAN, D. G. (1997). "Wage Differentials for Temporary Services Work: Evidence from Administrative Data". Working Paper Series. Research Department Federal reserve Bank of Chicago, No 98-23.

SMITH, R. S. (1990). "Mostly on Monday: Is workers' compensation covering off-the-job injuries?". En Philip S. Borba and David Appel (eds.): Benefits, Costs, and Cycles in Workers' Compensation. Boston: Kluwer Academic Publishers, 115-127.

SMITH, V. (2001). Teamwork versus Tempwork: Managers and The Dualism of Workplace Restructuring". En Cornfield, Campbell, McCammon (ed.): Working in Restructured Workplaces.

WARD, K., GRIMSHAW, D., RUBERY, J., y BEYNON, H. (2001). "Dilemmas in the Management of Temporary Work Agency Staff". Human Resource Management Journal, 11(4), 3-22.

YUN, M. (2004). "Decomposing differences in the first moment". Economics Letters, 82, 275-280.

YUN, M. (2005). "Normalized equation and decomposition analysis: computation and inference". IZA Discussion Paper, 1822, Tulane University. 


\section{Legislación}

- Estatuto de los Trabajadores.

- Ley 14/1994, de 1 de junio, por la que se regulan las Empresas de Trabajo Temporal.

- Real Decreto 4/1995, de 13 de enero, por el que se desarrolla la ley 14/1994, de 1 de junio, por la que se regulan las Empresas de Trabajo Temporal.

- Real Decreto 2720/1998, de 18 diciembre, por el que se desarrolla el artículo 15 del Estatuto de los Trabajadores en materia de contratos de duración determinada.

- Real Decreto 216/1999, de 5 de febrero, sobre disposiciones mínimas de Seguridad y Salud en el Trabajo en el Ámbito de las Empresas de Trabajo Temporal.

- Ley 29/1999, de 16 de julio, de Modificación de la Ley 14/1994, de 1 de junio, por la que se regulan las Empresas de Trabajo Temporal.

- Ley $14 / 2000$, de 29 de diciembre, de medidas fiscales, administrativas y del orden social.

- Ley $12 / 2001$, de 9 de julio, de medidas urgentes de reforma del mercado de trabajo para el incremento del empleo y la mejora de su calidad.

- Ley 35/2010, de 17 de septiembre, de medidas urgentes para la reforma del mercado de trabajo.

- Ley 3/2012 de 6 de julio, de medidas urgentes para la reforma del mercado laboral. 


\section{APÉNDICE 1}

Tabla A1

Resumen descriptivo de variables incluidas en el modelo (año 2006)

\begin{tabular}{|c|c|c|c|c|}
\hline & & ETT & TEMPORAL & INDEFINIDO \\
\hline \multicolumn{2}{|l|}{ Edad (años) } & 29.7 & 32.1 & 38.7 \\
\hline \multicolumn{2}{|c|}{ Antigüedad (meses) } & 2.2 & 9.7 & 81.9 \\
\hline \multicolumn{2}{|l|}{ Varón } & $70.28 \%$ & $69.07 \%$ & $73.60 \%$ \\
\hline \multicolumn{2}{|l|}{ Inmigrante } & $18.53 \%$ & $15.00 \%$ & $7.12 \%$ \\
\hline \multirow{9}{*}{ CNAE 93} & Agricultura, ganadería & $3.13 \%$ & $7.32 \%$ & $3.47 \%$ \\
\hline & Energía y agua & $0.33 \%$ & $0.85 \%$ & $0.76 \%$ \\
\hline & Industrias extractivas & $12.01 \%$ & $5.03 \%$ & $6.91 \%$ \\
\hline & Industria manufacturera & $26.91 \%$ & $17.26 \%$ & $17.12 \%$ \\
\hline & Otras industrias manufactureras & $22.96 \%$ & $11.84 \%$ & $15.24 \%$ \\
\hline & Comercio, reparación y hostelería & $13.95 \%$ & $27.15 \%$ & $29.40 \%$ \\
\hline & Transporte y comunicaciones & $11.53 \%$ & $9.06 \%$ & $9.51 \%$ \\
\hline & Instituciones financieras & $7.39 \%$ & $8.35 \%$ & $6.32 \%$ \\
\hline & Otros servicios & $1.78 \%$ & $13.13 \%$ & $11.27 \%$ \\
\hline \multirow{9}{*}{ CNO 94} & Directores, científicos e intelectuales & $0.08 \%$ & $0.07 \%$ & $0.37 \%$ \\
\hline & Profesionales de apoyo & $0.09 \%$ & $1.29 \%$ & $1.47 \%$ \\
\hline & Empleados de oficina & $0.63 \%$ & $2.64 \%$ & $3.20 \%$ \\
\hline & Restauración, comercio y salud & $1.57 \%$ & $3.15 \%$ & $4.21 \%$ \\
\hline & Protección y seguridad & $3.43 \%$ & $18.02 \%$ & $17.95 \%$ \\
\hline & Cualificados del sector $1^{\circ}$ y construcción & $0.65 \%$ & $2.56 \%$ & $2.28 \%$ \\
\hline & Cualificados de la industria & $10.65 \%$ & $22.13 \%$ & $24.98 \%$ \\
\hline & Operadores, instalaciones y conductores & $11.61 \%$ & $15.32 \%$ & $21.53 \%$ \\
\hline & No cualificados & $71.28 \%$ & $34.80 \%$ & $24.02 \%$ \\
\hline \multirow{17}{*}{$\begin{array}{l}\text { Comunidad } \\
\text { Autónoma }\end{array}$} & Andalucía & $7.30 \%$ & $21.23 \%$ & $12.20 \%$ \\
\hline & Aragón & $3.63 \%$ & $2.50 \%$ & $2.77 \%$ \\
\hline & Asturias & $1.44 \%$ & $2.17 \%$ & $1.74 \%$ \\
\hline & Baleares & $0.64 \%$ & $3.61 \%$ & $2.80 \%$ \\
\hline & Canarias & $1.70 \%$ & $5.05 \%$ & $4.52 \%$ \\
\hline & Cantabria & $2.19 \%$ & $0.94 \%$ & $1.14 \%$ \\
\hline & Castilla y León & $5.49 \%$ & $4.08 \%$ & $4.51 \%$ \\
\hline & Castilla La Mancha & $6.04 \%$ & $4.87 \%$ & $4.29 \%$ \\
\hline & Cataluña & $26.46 \%$ & $13.78 \%$ & $20.39 \%$ \\
\hline & Comunidad Valenciana & $10.85 \%$ & $10.06 \%$ & $10.57 \%$ \\
\hline & Extremadura & $0.75 \%$ & $1.76 \%$ & $1.25 \%$ \\
\hline & Galicia & $5.17 \%$ & $5.90 \%$ & $4.97 \%$ \\
\hline & Madrid & $13.67 \%$ & $14.04 \%$ & $16.80 \%$ \\
\hline & Murcia & $3.51 \%$ & $3.08 \%$ & $2.83 \%$ \\
\hline & Navarra & $2.10 \%$ & $1.20 \%$ & $1.79 \%$ \\
\hline & País Vasco & $8.40 \%$ & $5.20 \%$ & $6.63 \%$ \\
\hline & La Rioja & $0.66 \%$ & $0.53 \%$ & $0.79 \%$ \\
\hline \multicolumn{2}{|c|}{ Accidentes de difícil diagnóstico } & $38.31 \%$ & $41.10 \%$ & $45.13 \%$ \\
\hline \multicolumn{2}{|c|}{ ACCIDENTES } & 22,481 & 246,489 & 356,666 \\
\hline
\end{tabular}

Fuente: Elaboración propia a partir de datos de la EAT para el año 2006. 
Tabla A2

Resumen descriptivo de variables incluidas en el modelo (año 2011)

\begin{tabular}{|c|c|c|c|c|}
\hline & & ETT & TEMPORAL & INDEFINIDO \\
\hline \multicolumn{2}{|l|}{ Edad (años) } & 32.8 & 35.5 & 41 \\
\hline \multicolumn{2}{|l|}{ Antigüedad (meses) } & 2.4 & 14.3 & 94.1 \\
\hline \multicolumn{2}{|l|}{ Varón } & $70.95 \%$ & $62.07 \%$ & $65.79 \%$ \\
\hline \multicolumn{2}{|l|}{ Inmigrante } & $19.15 \%$ & $13.24 \%$ & $8.24 \%$ \\
\hline \multirow{8}{*}{ CNAE 2009} & Agricultura, ganadería & $12.37 \%$ & $10.68 \%$ & $2.83 \%$ \\
\hline & Energía y agua e Industrias extractivas & $0.79 \%$ & $2.81 \%$ & $3.23 \%$ \\
\hline & Industria manufacturera & $20.47 \%$ & $5.18 \%$ & $8.02 \%$ \\
\hline & Otras industrias manufactureras & $28.07 \%$ & $12.07 \%$ & $16.84 \%$ \\
\hline & Comercio, reparación y hostelería & $15.26 \%$ & $24.32 \%$ & $29.55 \%$ \\
\hline & Transporte y comunicaciones & $10.82 \%$ & $7.69 \%$ & $9.79 \%$ \\
\hline & Instituciones financieras & $8.12 \%$ & $3.65 \%$ & $3.56 \%$ \\
\hline & Otros servicios & $4.10 \%$ & $33.60 \%$ & $26.18 \%$ \\
\hline \multirow{9}{*}{ CNO 2011} & Directores, científicos e intelectuales & $0.29 \%$ & $3.71 \%$ & $3.76 \%$ \\
\hline & Profesionales de apoyo & $1.35 \%$ & $3.51 \%$ & $3.66 \%$ \\
\hline & Empleados de oficina & $3.36 \%$ & $4.02 \%$ & $5.39 \%$ \\
\hline & Restauración, comercio y salud & $4.65 \%$ & $21.53 \%$ & $21.09 \%$ \\
\hline & Protección y seguridad & $0.04 \%$ & $1.44 \%$ & $4.59 \%$ \\
\hline & Cualificados del sector $1^{\circ}$ y construcción & $2.63 \%$ & $7.67 \%$ & $5.31 \%$ \\
\hline & Cualificados de la industria & $11.59 \%$ & $10.60 \%$ & $16.38 \%$ \\
\hline & Operadores, instalaciones y conductores & $12.64 \%$ & $10.85 \%$ & $16.66 \%$ \\
\hline & No cualificados & $63.47 \%$ & $36.65 \%$ & $23.14 \%$ \\
\hline \multirow{17}{*}{ Comunidad Autónoma } & Andalucía & $8.30 \%$ & $22.48 \%$ & $13.58 \%$ \\
\hline & Aragón & $3.78 \%$ & $2.48 \%$ & $2.77 \%$ \\
\hline & Asturias & $2.50 \%$ & $2.66 \%$ & $2.55 \%$ \\
\hline & Baleares & $0.50 \%$ & $3.69 \%$ & $2.99 \%$ \\
\hline & Canarias & $2.40 \%$ & $6.35 \%$ & $4.77 \%$ \\
\hline & Cantabria & $1.49 \%$ & $0.98 \%$ & $1.17 \%$ \\
\hline & Castilla y León & $3.99 \%$ & $4.64 \%$ & $4.76 \%$ \\
\hline & Castilla La Mancha & $3.64 \%$ & $4.37 \%$ & $3.74 \%$ \\
\hline & Cataluña & $24.35 \%$ & $12.72 \%$ & $18.88 \%$ \\
\hline & Comunidad Valenciana & $14.26 \%$ & $7.73 \%$ & $8.72 \%$ \\
\hline & Extremadura & $0.67 \%$ & $2.67 \%$ & $1.40 \%$ \\
\hline & Galicia & $6.74 \%$ & $6.20 \%$ & $5.31 \%$ \\
\hline & Madrid & $8.76 \%$ & $12.81 \%$ & $17.99 \%$ \\
\hline & Murcia & $8.11 \%$ & $2.87 \%$ & $2.62 \%$ \\
\hline & Navarra & $2.64 \%$ & $1.38 \%$ & $1.70 \%$ \\
\hline & País Vasco & $7.41 \%$ & $5.44 \%$ & $6.24 \%$ \\
\hline & La Rioja & $0.45 \%$ & $0.54 \%$ & $0.79 \%$ \\
\hline \multicolumn{2}{|c|}{ Accidentes de difícil diagnóstico } & $44.08 \%$ & $48.49 \%$ & $51.44 \%$ \\
\hline \multicolumn{2}{|l|}{ ACCIDENTES } & 8,069 & 127,784 & 303,224 \\
\hline
\end{tabular}

Fuente: Elaboración propia a partir de datos de la EAT para el año 2011. 


\section{Tabla A3}

Estimaciones de la probabilidad de que un accidente sufrido sea de difícil diagnóstico por tipo de contrato (año 2006)

\begin{tabular}{|c|c|c|c|c|c|c|}
\hline \multirow[b]{2}{*}{ Varón } & \multicolumn{2}{|c|}{ ETT } & \multicolumn{2}{|c|}{ Temporal } & \multicolumn{2}{|c|}{ Indefinido } \\
\hline & -0.1659 & *** & -0.1642 & $* \star *$ & -0.1424 & *** \\
\hline Edad & 0.0209 & 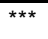 & 0.0207 & 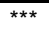 & 0.0189 & 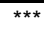 \\
\hline Edad al cuadrado & -0.0003 & $\star \star \star *$ & -0.0003 & $\star \star \star *$ & -0.0003 & *** \\
\hline Antigüedad & 0.0031 & & 0.0022 & 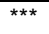 & 0.0008 & 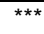 \\
\hline Antigüedad al cuadrado & $-1.55 E-05$ & & $-5.08 \mathrm{E}-06$ & $\star \star \star *$ & $-1.48 \mathrm{E}-06$ & 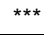 \\
\hline Inmigrante (desarrollado) & -0.0285 & & -0.0830 & 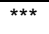 & -0.1278 & *** \\
\hline Inmigrante (no desarrollado) & -0.2239 & 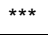 & -0.2074 & $\star \star \star *$ & -0.1887 & 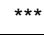 \\
\hline Lunes & 0.0666 & $* \star *$ & 0.0637 & $* \star *$ & 0.0768 & *** \\
\hline Fin de semana & 0.0449 & & -0.0300 & $\star * \star$ & -0.0471 & $\star * *$ \\
\hline En otro centro & 0.0860 & * & 0.0161 & & 0.0394 & \\
\hline Desplazamiento en jornada & -0.6719 & $\star \star$ & -0.1514 & $\star \star$ & -0.0716 & ** \\
\hline Ir o volver del trabajo & -0.1643 & 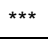 & -0.0320 & $\star \star$ & -0.0402 & \\
\hline Entre la $2^{\mathrm{a}}$ y $8^{\mathrm{a}}$ hora & -0.0781 & 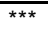 & -0.0414 & 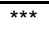 & -0.0465 & 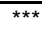 \\
\hline Más de 8 horas trabajadas & -0.1410 & $\star \star$ & -0.0231 & & -0.0432 & $\star \star *$ \\
\hline Mañana & 0.0726 & 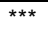 & 0.0579 & 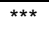 & 0.0573 & 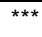 \\
\hline Tarde & 0.0181 & & 0.0014 & & -0.0036 & \\
\hline Org. Preventiva: Personal & -0.1424 & & 0.0329 & * & -0.0133 & *** \\
\hline Org. Preventiva: Trabajador designado & -0.2161 & 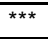 & 0.0303 & * & 0.0264 & ** \\
\hline Org. Preventiva: Servicio propio & -0.0824 & $* \star \star \star \Delta$ & 0.0461 & $* \star \star$ & 0.0253 & 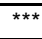 \\
\hline Org. Preventiva: Servicio mancomunado & -0.0366 & & 0.0439 & 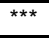 & 0.0012 & ** \\
\hline Org. Preventiva: Servicio contratado & 0.0100 & & 0.0041 & & -0.0321 & *** \\
\hline Evaluación de riesgos & 0.0954 & 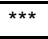 & 0.0089 & & 0.0057 & 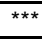 \\
\hline Profesionales de apoyo & -0.0006 & & 0.0815 & & -0.0562 & 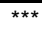 \\
\hline Empleados de oficina & 0.0376 & & 0.2608 & $\star \star \star *$ & 0.0810 & \\
\hline Restauración comercio y salud & 0.0827 & & 0.1729 & * & -0.0227 & \\
\hline Servicios, protección y seguridad & -0.0639 & & 0.0656 & & -0.0761 & \\
\hline Cualificados sector $1^{\circ}$ & 0.1820 & & 0.0868 & & -0.0562 & $\star \star \star *$ \\
\hline Cualificados de la industria & 0.0023 & & 0.0048 & & -0.1324 & *** \\
\hline Operadores, instaladores, conductores & -0.0565 & & 0.0927 & & -0.0648 & \\
\hline No cualificados & -0.0084 & & 0.0970 & & -0.0424 & * \\
\hline Energía, agua & -0.1848 & & -0.0138 & & 0.0661 & \\
\hline industrias extractivas & -0.0822 & & -0.1139 & $* * *$ & -0.0337 & \\
\hline Industria manufacturera & -0.1876 & $\star \star *$ & -0.2191 & $\star * \star$ & -0.1232 & $* * *$ \\
\hline Otras industrias manufactureras & -0.0322 & & -0.1423 & $\star \star *$ & -0.1089 & $* \star *$ \\
\hline Comercio, reparación y hostelería & 0.0144 & & -0.0605 & $\star \star \star ~$ & -0.0030 & *** \\
\hline Transporte y comunicaciones & 0.1195 & $\star \star$ & 0.0827 & $\star \star \star *$ & 0.1651 & \\
\hline Instituciones financieras & -0.0918 & & 0.0775 & $\star * \star$ & 0.1205 & *** \\
\hline Otros servicios & 0.2388 & $* * *$ & 0.1573 & $* * *$ & 0.1645 & *** \\
\hline 11 a 25 trabajadores & 0.0071 & & 0.0315 & 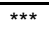 & 0.0250 & $* \star \star *$ \\
\hline 26 a 50 trabajadores & -0.0126 & & 0.0364 & $* * *$ & 0.0640 & *** \\
\hline 51 a 100 trabajadores & 0.0262 & & 0.0885 & $* * *$ & 0.0966 & $* * *$ \\
\hline 101 a 250 trabajadores & 0.0573 & * & 0.1285 & $* * *$ & 0.1498 & *** \\
\hline 251 a 500 trabajadores & 0.1967 & 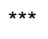 & 0.1088 & $* \star *$ & 0.1532 & *** \\
\hline Más de 500 trabajadores & 0.0546 & & 0.0998 & $\star \star \star$ & 0.1243 & $\star \star \star$ \\
\hline Constante & -0.5480 & & -0.5331 & $\star \star \star *$ & -0.3195 & 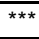 \\
\hline
\end{tabular}

*, ** $y{ }^{* \star *}$ Indican significación al 10\%, 5\%, y 1\% respectivamente.

Nota: Además de todas las variables que aparecen en la tabla se han incluido controles de Comunidad Autónoma.

Fuente: Elaboración propia a partir de datos de la EAT para el año 2006. 
Tabla A4

Estimaciones de la probabilidad de que un accidente sufrido sea de difícil diagnóstico por tipo de contrato (año 2011)

\begin{tabular}{|c|c|c|c|c|c|c|}
\hline \multirow[b]{2}{*}{ Varón } & \multicolumn{2}{|c|}{ ETT } & \multicolumn{2}{|c|}{ Temporal } & \multicolumn{2}{|c|}{ Indefinido } \\
\hline & -0.1970 & 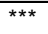 & -0.1752 & *** & -0.1387 & 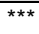 \\
\hline Edad & -0.0122 & & 0.0166 & *** & 0.0194 & *** \\
\hline Edad al cuadrado & 0.0001 & & -0.0003 & $\star \star *$ & -0.0003 & *** \\
\hline Antigüedad & 0.0047 & & 0.0020 & 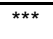 & 0.0006 & 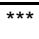 \\
\hline Antigüedad al cuadrado & 2.5E-05 & & $-4.65 E-06$ & $\star * \star$ & $-9.07 \mathrm{E}-07$ & *** \\
\hline Inmigrante (desarrollado) & 0.0603 & & -0.1133 & *** & -0.1565 & 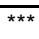 \\
\hline Inmigrante (no desarrollado) & -0.2067 & $\star * \star$ & -0.1850 & 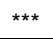 & -0.1662 & *** \\
\hline Lunes & 0.0280 & & 0.0632 & $* \star \star *$ & 0.0789 & *** \\
\hline Fin de semana & -0.0060 & & -0.0345 & $\star * \star$ & -0.0471 & $\star \star \star *$ \\
\hline En otro centro & 0.1097 & & 0.0483 & $* \star \star$ & 0.0135 & 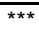 \\
\hline Desplazamiento en jornada & 0.3608 & $\star \star \star *$ & 0.0548 & $\star \star \star *$ & -0.0231 & \\
\hline Ir o volver del trabajo & -0.1346 & $\star \star$ & 0.0037 & & 0.0212 & *** \\
\hline Entre la $2^{\mathrm{a}}$ y $8^{\mathrm{a}}$ hora & 0.0334 & & -0.0530 & $* \star * *$ & -0.0602 & *** \\
\hline Más de 8 horas trabajadas & 0.0957 & & -0.0013 & & -0.0464 & ** \\
\hline Mañana & -0.0044 & & 0.0501 & $* \star \star *$ & 0.0613 & 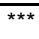 \\
\hline Tarde & -0.0714 & * & -0.0056 & & -0.0105 & \\
\hline Org. Preventiva: Personal & 0.3737 & ** & 0.0904 & 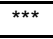 & 0.0816 & \\
\hline Org. Preventiva: Trabajador designado & 0.2719 & 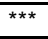 & 0.0061 & & -0.0309 & ** \\
\hline Org. Preventiva: Servicio propio & 0.0601 & & 0.0462 & 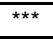 & 0.0536 & 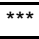 \\
\hline Org. Preventiva: Servicio mancomunado & 0.0441 & & 0.0524 & *** & 0.0213 & \\
\hline Org. Preventiva: Servicio contratado & 0.2053 & 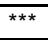 & 0.0865 & 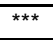 & 0.0721 & 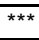 \\
\hline Evaluación de riesgos & 0.0043 & & 0.0164 & ** & 0.0307 & \\
\hline Profesionales de apoyo & -0.1164 & & 0.1678 & *** & 0.0870 & \\
\hline Empleados de oficina & 0.1415 & & -0.0325 & & 0.0139 & ** \\
\hline Restauración comercio y salud & -0.0080 & & -0.0377 & * & -0.0109 & \\
\hline Servicios, protección y seguridad & -0.4503 & & -0.0378 & & 0.0090 & ** \\
\hline Cualificados sector $1^{\circ}$ & 0.3587 & & -0.0789 & $\star \star \star *$ & -0.0771 & \\
\hline Cualificados de la industria & 0.1053 & & -0.1507 & $\star * *$ & -0.1057 & *** \\
\hline Operadores, instaladores, conductores & 0.0970 & & -0.0384 & * & -0.0006 & * \\
\hline No cualificados & 0.0716 & & -0.0437 & $\star \star$ & -0.0236 & \\
\hline Energía, agua e industrias extractivas & -0.3956 & ** & -0.0601 & ** & -0.0284 & ** \\
\hline Industria manufacturera & -0.2533 & $* * *$ & -0.1804 & $\star \star \star *$ & -0.0811 & ** \\
\hline Otras industrias manufactureras & -0.2294 & $\star \star \star *$ & -0.1983 & $* * *$ & -0.1012 & *** \\
\hline Comercio, reparación y hostelería & -0.0592 & & -0.1343 & $\star \star * *$ & -0.0119 & *** \\
\hline Transporte y comunicaciones & 0.1914 & $\star \star$ & 0.0430 & $\star *$ & 0.1351 & \\
\hline Instituciones financieras & -0.1437 & * & 0.0453 & $\star \star$ & 0.0517 & *** \\
\hline Otros servicios & 0.0213 & & 0.1224 & $\star \star \star *$ & 0.1302 & *** \\
\hline 11 a 25 trabajadores & -0.0544 & & 0.0538 & 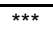 & 0.0299 & 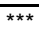 \\
\hline 26 a 50 trabajadores & 0.1281 & * & 0.0768 & $\star * \star$ & 0.0723 & *** \\
\hline 51 a 100 trabajadores & 0.1581 & $\star * \star$ & 0.1357 & $\star * *$ & 0.1031 & *** \\
\hline 101 a 250 trabajadores & 0.2203 & $\star * \star$ & 0.1481 & $* * *$ & 0.1337 & *** \\
\hline 251 a 500 trabajadores & 0.1004 & & 0.1428 & $\star * *$ & 0.1029 & *** \\
\hline Más de 500 trabajadores & 0.2006 & $\star *$ & 0.1060 & $\star \star \star *$ & 0.0858 & *** \\
\hline Constante & -0.0451 & & -0.2715 & *** & -0.3639 & 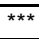 \\
\hline
\end{tabular}

*, ** $y^{* * *}$ Indican significación al 10\%, 5\%, y 1\% respectivamente.

Nota: Además de todas las variables que aparecen en la tabla se han incluido controles de Comunidad Autónoma.

Fuente: Elaboración propia a partir de datos de la EAT para el año 2011. 